\title{
Gauge Block Calibration with Very SMALl MEASUREMENT UNCERTAINTY
}

\author{
GODINA, A. \& ACKO, B.
}

Abstract: For a national metrology laboratory and holder of national standard for length, capability of performing gauge block calibration by mechanical comparison with lowest possible uncertainty is of highest importance. Uncertainty of gauge block calibration at national metrology laboratory namely enters budgets of uncertainty evaluation of all industrial length calibration laboratories throughout the country.

In present paper the uncertainty budget of mechanical calibration of gauge blocks, as a results of extensive analytical and experimental research, is presented in details. Very low measurement uncertainty was achieved and procedure was accredited and entered as Calibration and measurement capability (CMC) into the key comparison database at BIPM.

Key words: gauge block, calibration, mechanical comparison, measurement uncertainty
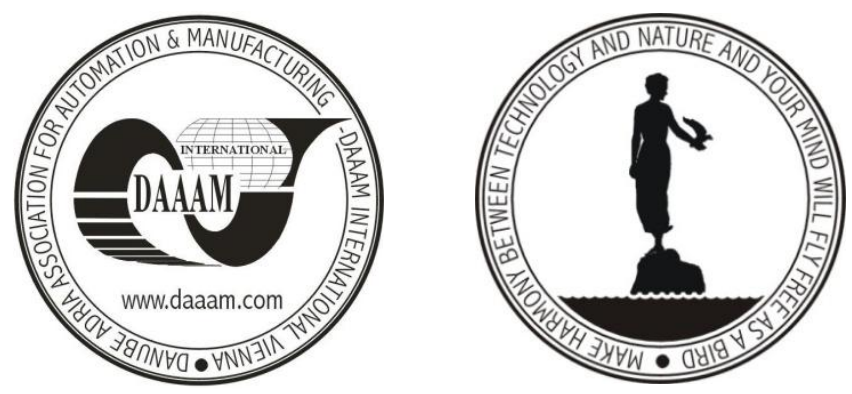

Authors' data: Dr. Godina, A[ndrej]; Prof. Dr. Acko, B[ojan], University of Maribor, Faculty of mechanical engineering, Smetanova 17, 2000 Maribor, Slovenia, andrej.godina@um.si, bojan.acko@um.si

This Publication has to be referred as: Godina, A[ndrej] \& Acko, B[ojan] (2013) Gauge Block Calibration with Very Small Measurement Uncertainty, Chapter 16 in DAAAM International Scientific Book 2013, pp. 339-350, B. Katalinic \& Z. Tekic (Eds.), Published by DAAAM International, ISBN 978-3-901509-94-0, ISSN 17269687, Vienna, Austria

DOI: $10.2507 /$ daaam.scibook.2013.16 
Godina, A. \& Acko, B.: Gauge Block Calibration with Very Small Measurement Un..

\section{Introduction}

Gauge blocks are being calibrated by two methods: interferometric calibration and calibration by mechanical comparison to reference gauge block. While interferometric method represents primary level, comparison is more suitable for calibrating industrial gauge blocks because of lower costs and shorter calibration time. Although no significant changes in equipment happened in last decade, improvements in mechanical comparison are on-going (Godina et al., 2006). A short review of the Calibration and Measurement capabilities - CMC (***a) shows that the ratio between high- and low-end uncertainties in mechanical calibration is approx. 1:3. That indicates the potential for further improvement in lowering the uncertainty.

Especially for national metrology laboratories, not performing interferometric gauge block calibration, capability of performing gauge block calibration by mechanical comparison with lowest possible uncertainty is of highest importance (Acko, 2012). Uncertainty of gauge block calibration at national metrology laboratory namely enters budgets of uncertainty evaluation of all industrial length calibration laboratories throughout the country.

\section{Calibration Laboratory as a Holder of a National Standard}

As a part of distributed system of national measurement standards, Laboratory for Production Measurement (LTM) at the Faculty for mechanical engineering, University of Maribor, is maintaining the national standard for length since 1998. Since the uncertainty of measurement in calibrations performed in LTM enters into the uncertainty budgets of calibration laboratories throughout the country, the uncertainty minimization is one of our highest ranked fields of research.

National standard for length in Slovenia comprise of 122-piece set of steel gauge blocks, ranging from $0,5 \mathrm{~mm}$ to $125 \mathrm{~mm}$ and some additional steel gauge blocks of length up to $1000 \mathrm{~mm}$. These gauge blocks will be referred to as reference gauge blocks.

Reference gauge blocks are calibrated externally in European highest rank metrological institution by primary standard. In the case of length that is interferometric method.

\section{Length Standard: Gauge Block}

Gauge blocks are most accurate standards of length and an important basis of industrial length measurements, as well as the most important and commonly used measurement standards for maintaining traceability in dimensional metrology (Faust et al., 1998). They provide industry with reliable and traceable standards of length.

Gauge blocks are defined in ISO standard (ISO 3650, 1998). Gauge block is length standard of rectangular shape, made of wearing resistant material. It comprises of pair of flat, mutually parallel measurement surfaces, which can be wrung to 
measurement surfaces of other gauge blocks and herewith joined (see Fig. 1). Gauge block can also be wrung onto wringing plate with similar surface quality, what is used at interferometric length measurement.

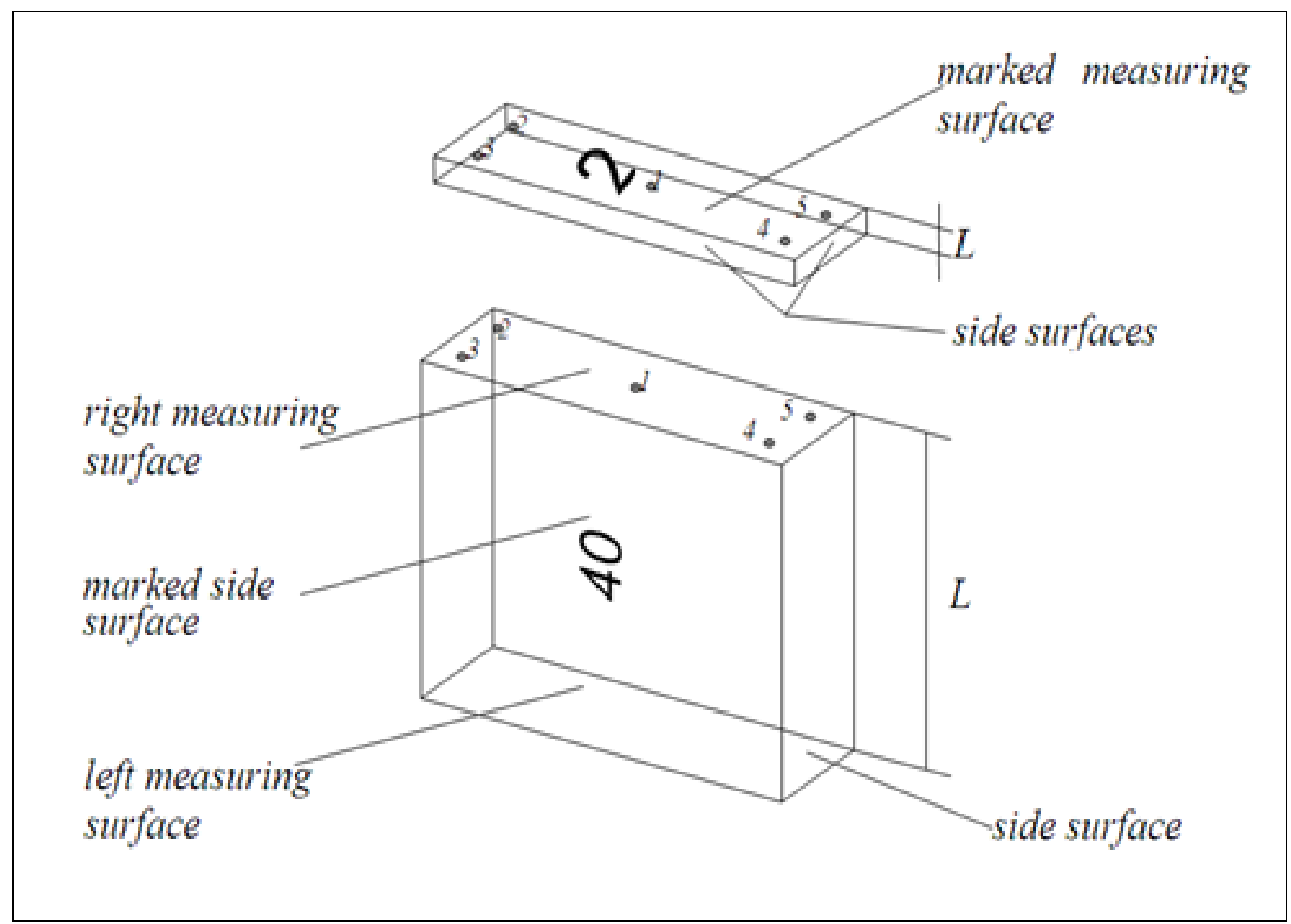

Fig. 1. Gauge blocks

Depending of gauge block length, two different dimensions of measuring surfaces exist (see Tab. 1).

\begin{tabular}{|c|c|}
\hline Gauge block's nominal length $L$ in $\mathrm{mm}$ & Measuring surfaces dimension in mm \\
\hline $0,5 \leq L<10$ & $30 \times 9$ \\
\hline$L \geq 10$ & $35 \times 9$ \\
\hline
\end{tabular}

Tab. 1. Gauge block's measuring surfaces dimensions

On a gauge block, nominal dimension, name or sign of the manufacturer and identification number are marked. Gauge blocks of dimension $\mathrm{L}<6 \mathrm{~mm}$ are marked on the measuring surfaces, as shown in Fig. 1. 
Godina, A. \& Acko, B.: Gauge Block Calibration with Very Small Measurement Un..

\section{Gauge Block Comparator}

For calibration of gauge blocks of length up to $125 \mathrm{~mm}$ by mechanical comparison a contact comparator is used. Typical gauge block comparator (in our case Mahr 826) comprises of the measurement pedestal, the measurement table with the gauge block positioning device, two length indicators (probe A and B) connected to an electronic measuring instrument with numerical display (Mahr, 1995) (Fig. 2).

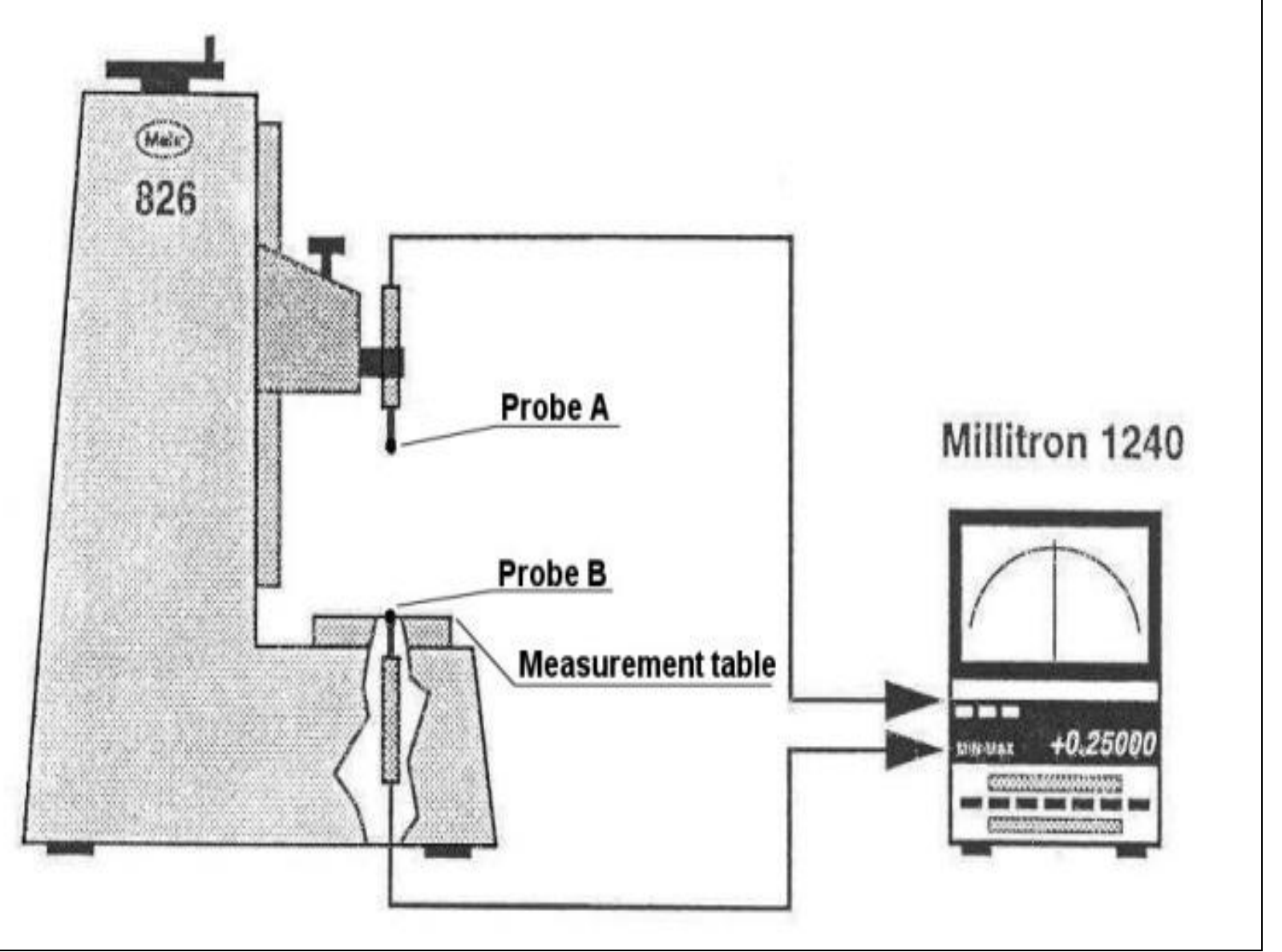

Fig. 2. Gauge block comparator (Mahr)

\section{Calibration of Gauge Blocks by Mechanical Comparison}

\subsection{Preparation for Calibration}

Before calibration, gauge blocks must be carefully cleaned and stored in the microclimatic chamber for at least 24 hours in order to get right temperature (Thalmann et al., 2003). Microclimatic conditions should be stabile temperature in the range of $(20 \pm 0.3){ }^{\circ} \mathrm{C}$, actual temperature in the moment of probing enters into the measuring programme in order to calculate the temperature expansion correction.

\subsection{Performning the Measurement}

The measurement is supported by software, provided by comparator's manufacturer. In accordance with (ISO 3650, 1998), for the highest level measurements five points are measured. The procedure is divided in the following steps (Godina et al., 2007): 
- Start of the measuring programme, entering measured temperature value;

- Measurement of the midpoint of the gauge block A; resetting a display to $0.00 \mu \mathrm{m}$;

- Moving the gauge blocks to measure the midpoint of the gauge block B (measuring point No. 1 - see Fig. 1). The point should be probed at least three times;

- Measurements in the points 2, 3,4 and 5. Each point should be probed at least three times,

- Repeated measurement in the midpoint of the gauge block A: results of repeated measurements should lie in the tolerance of $0,02 \mu \mathrm{m}$ (otherwise the measurement is not valid and must be repeated).

\section{Evaluation of Measurement Results}

\subsection{Correction of Thermal Expansion}

If gauge blocks $\mathrm{A}$ and $\mathrm{B}$ are made of equal materials, the expansions caused by temperature deviation $\Delta t$ (reference temperature is $20^{\circ} \mathrm{C}$ ) are equal. Therefore, a temperature expansion correction is not calculated.

\subsection{Calculation of the Gauge Block's Deviation from the Nominal Value}

Mean value (indication) of the gauge block A:

$$
A_{o}=\frac{1}{2}\left(A_{o_{a}}+A_{o_{b}}\right)
$$

Difference between lengths of gauge blocks A and B in the measuring point x:

$$
\Delta_{x}=B_{x}-A_{o}
$$

Deviation of the gauge block B from the nominal value in the measuring point at $20{ }^{\circ} \mathrm{C}$ :

$$
(d e v) B_{x}=\Delta_{x}+(d e v) A
$$

where:

$(d e v) B_{x^{-}}$indicated value in the measuring point x;

(dev)A - deviation of the gauge block A from the nominal value in point 1 (as determined by calibration of the reference gauge blocks).

\section{Measurement Uncertainty Analysis}

Calibration uncertainty analysis follows ISO Guide to the expression of uncertainty in measurement (ISO Guide, 1995), as well as European Accreditation publication Expressions of the Uncertainty of Measurements in Calibration (EA-4/02, 1999). 
Godina, A. \& Acko, B.: Gauge Block Calibration with Very Small Measurement Un..

\subsection{Mathematical Model Of the Measurement}

The length $L_{x}$ of the gauge block being calibrated is given by the expression:

$$
L_{x}=L_{s}+\delta L_{d}+\delta L+\delta L_{c}-L\left(\bar{\alpha} \cdot \delta t+\delta \alpha_{x} \cdot \Delta \bar{t}\right)-\delta L_{V}
$$

where:

$$
\begin{gathered}
\delta t=\left(t_{x}-t_{s}\right) \\
\bar{\alpha}=\left(\alpha_{x}+\alpha_{s}\right) / 2
\end{gathered}
$$

$L_{\mathrm{s}}$ - length of the reference gauge block at the reference temperature $t_{0}=20{ }^{\circ} \mathrm{C}$ according to its calibration certificate;

$\delta L_{\mathrm{D}}$ - change of the length of the reference gauge block since its last calibration due to drift;

$\delta L$ - observed difference in length between the measured and the reference gauge block;

$\delta L_{C^{-}}$correction for non-linearity of the comparator;

$L$ - nominal length of the gauge blocks considered;

$\alpha_{\mathrm{s}}$, - thermal expansion coefficients of the reference gauge block;

$\alpha_{x}$ - thermal expansion coefficients of the measured gauge block;

$\bar{\alpha}$ - average of thermal expansion coefficients;

$t_{s}$ - temperature of the reference gauge block;

$t_{x}$ - temperature of the measured gauge block;

$\delta t$ - temperature difference between both gauge blocks;

$\delta L_{V}$ - correction for non-central contacting of the measuring faces of the measured gauge block.

\subsection{Standard Uncertainties of the Input Values Estimations for Calculating the Combined Standard Uncertainty}

Combined standard uncertainty is expressed by the uncertainties of the input values by the following equation:

$$
\begin{aligned}
& u_{\mathrm{S}}^{2}\left(L_{\mathrm{x}}\right)={c_{L_{\mathrm{s}}}}^{2} u^{2}\left(L_{\mathrm{s}}\right)+c_{\delta L_{D}}{ }^{2} u^{2}\left(\delta L_{D}\right)+c_{\delta L}{ }^{2} u^{2}(\delta L)+c_{\delta L_{C}}{ }^{2} u^{2}\left(\delta L_{C}\right)+ \\
& +c_{\bar{\alpha}}{ }^{2} u^{2}(\bar{\alpha})+c_{\delta t}{ }^{2} u^{2}(\delta t)+c_{\partial \alpha}{ }^{2} u^{2}(\delta \alpha)+c_{\Delta \bar{t}}{ }^{2} u^{2}(\Delta \bar{t})+c_{\delta L_{V}}{ }^{2} u^{2}\left(\delta L_{V}\right)
\end{aligned}
$$

where $c_{i}$ are partial derivatives of the function (4):

$$
\mathrm{c}_{L_{s}}=\partial f / \partial L_{s}=1
$$




$$
\begin{gathered}
c_{\delta L_{D}}=\partial f / \partial \delta L_{D}=1 \\
c_{\delta L}=\partial f / \partial L=1 \\
c_{\delta L_{C}}=\partial f / \partial L_{C}=1 \\
c_{\bar{\alpha}}=\partial f / \partial L_{\bar{\alpha}}=-L \delta t \\
c_{\partial t}=\partial f / \partial L_{\delta t}=-L \bar{\alpha} \\
c_{\partial \alpha}=\partial \mathrm{f} / \partial \mathrm{L}_{\delta \alpha}=-\mathrm{L} \Delta \overline{\mathrm{t}} \\
c_{\Delta \bar{t}}=\partial f / \partial L_{\Delta \bar{t}}=-L \delta \alpha \\
c_{\delta L_{V}}=\partial f / \partial L_{s \delta L_{V}}=-1
\end{gathered}
$$

Standard uncertainties of the input values are evaluated (estimated) for the applied equipment and method as well as for supposed measurement conditions.

\subsubsection{Uncertainty of the reference standard length $u\left(L_{s}\right)$}

The uncertainty of the reference gauge block calibration is given in the calibration certificate of the set of gauge blocks as

$$
U=\sqrt{(20 \mathrm{~nm})^{2}+\left(0.18 \cdot 10^{-6} \cdot L\right)^{2}}
$$

Coverage factor $k=2$.

Standard uncertainty is therefore:

$$
u=\sqrt{(10 \mathrm{~nm})^{2}+\left(0.09 \cdot 10^{-6} \cdot L\right)^{2}}
$$

\subsubsection{Uncertainty caused by the drift of the standard $u\left(\delta L_{D}\right)$}

The temporal drift of the length of the reference gauge block is estimated from previous calibrations to be zero with limits $\pm 0,2 \cdot 10^{-6} \cdot \mathrm{L}$ for the recalibration period of two years. General experience with gauge blocks of this type suggests that zero drift is most probable and that a triangular probability distribution can be assumed.

Standard uncertainty is therefore:

$$
u=0,2 \cdot 10^{-6} \cdot L / \sqrt{6}=0,082 \cdot 10^{-6} \cdot L
$$


Godina, A. \& Acko, B.: Gauge Block Calibration with Very Small Measurement Un..

7.2.3 Uncertainty of the observed difference in length between the measured and the reference $G B u(\delta L)$

The measured difference can be expressed by the equation:

$$
\delta L=r-e_{S}
$$

where:

$\delta L$ - observed difference in length;

$r$ - reading;

$e_{\mathrm{s}}$ - offset of the comparator, as observed during calibration.

The uncertainty of the observed difference in length is therefore:

$$
u(\delta L)=\sqrt{u(r)^{2}+u\left(e_{s}\right)^{2}}
$$

The uncertainty of the reading can be expressed from the known interval in which the result is rounded. The comparator resolution is $10 \mathrm{~nm}$, therefore the interval of rounding is $\pm 5 \mathrm{~nm}$. Since the distribution is rectangular, the standard uncertainty is:

$$
u(r)=5 / \sqrt{3}=2.89 \mathrm{~nm}
$$

The uncertainty of the offset evaluation is stated in the calibration report. The comparator was calibrated in-house. The uncertainty of the calibration is:

$$
U=20 \mathrm{~nm}+0,2 \cdot 10^{-6} \cdot L ; k=2
$$

Standard uncertainty is therefore:

$$
u=10 \mathrm{~nm}+0,1 \cdot 10^{-6} \cdot L
$$

This formula can be expressed in quadratic form by considering the uncertainties on the lower and the upper measurement range limits $(0,5 \mathrm{~mm}$ and 100 $\mathrm{mm})$ :

$$
u(c a l)=\sqrt{(10 \mathrm{~nm})^{2}+\left(0.17 \cdot 10^{-6} \cdot L\right)^{2}}
$$

Total uncertainty of the observed difference in length is:

$$
u(\delta L)=\sqrt{(10,5 n m)^{2}+\left(0.17 \cdot 10^{-6} \cdot L\right)^{2}}
$$


The offset itself is not corrected during calibration of the gauge blocks, but is not allowed to exceed the resolution during the calibration of the comparator (in such case the probes should be tested and replaced if necessary).

\subsubsection{Uncertainty of the correction for non-linearity of the comparator $u\left(\delta L_{c}\right)$}

Taking into account the tolerances of the grade 0 measured gauge block and the grade $\mathrm{K}$ reference gauge block, the maximum length difference will be within $\pm 1,8 \mu \mathrm{m}$, leading to unidentifiable limits for the non-linearity of the comparator used (Godina et al., 2010).

\subsubsection{Uncertainty of temperature expansion coefficient $u(\bar{\alpha})$}

Experience values $\pm 0,5 \cdot 10^{-6}{ }^{\circ} \mathrm{C}^{-1}$ and rectangular distribution (equal possibility over the entire interval) are assumed. Standard uncertainty is therefore:

$$
u(\bar{\alpha})=\left(0,5 \cdot 10^{-6}{ }^{\circ} \mathrm{C}^{-1}\right) / \sqrt{3}=0,289 \cdot 10^{-6}{ }^{\circ} \mathrm{C}^{-1}
$$

\subsubsection{Uncertainty of temperature difference between the unknown and reference} gauge blocks $u(\delta t)$

Concerning the temperature measurements in the entire measuring space it can be assumed that the difference in temperatures of GBs lies with an equal probability in an interval $\pm 0,02{ }^{\circ} \mathrm{C}$.

The standard uncertainty is therefore:

$$
u(\delta t)=\left(0,02{ }^{\circ} \mathrm{C}\right) / \sqrt{3}=0,0115^{\circ} \mathrm{C}
$$

\subsubsection{Uncertainty of temperature expansion coefficient difference $u(\delta \alpha)$}

Interval of the difference is assumed according to the uncertainties of separate coefficients. It is $\pm 1 \cdot 10-6{ }^{\circ} \mathrm{C}-1$. Standard uncertainty at supposed triangular distribution is therefore:

$$
u(\delta \alpha)=\left(1 \cdot 10^{-6}{ }^{\circ} C^{-1}\right) / \sqrt{6}=0,41 \cdot 10^{-6}{ }^{\circ} C^{-1}
$$

7.2.8 Uncertainty of the deviation of GBs average temperature from the reference temperature $u(\Delta \bar{t})$

- Uncertainty of the temperature measurement system $u\left(\theta_{1}\right)$

The calibration certificate gives an uncertainty of $U=5 \mathrm{mK}$ with $k=2$. Standard uncertainty is therefore:

$$
u\left(\theta_{l}\right)=5 \cdot 10^{-3} / 2=0,0025^{\circ} \mathrm{C}
$$


Godina, A. \& Acko, B.: Gauge Block Calibration with Very Small Measurement Un..

- Uncertainty because of the difference between the table temperature and the GBs mean temperature

The difference between the table temperature and GBs mean temperature, as calculated from 80 measurements, was $0,025{ }^{\circ} \mathrm{C}$ with the standard deviation of 0.022 ${ }^{\circ} \mathrm{C}$. This difference is assumed to be a random error and contributes to the uncertainty. The total uncertainty is:

$$
u(\Delta \theta)=\sqrt{0,025^{2}+0,022^{2}}=0,033^{\circ} \mathrm{C}
$$

- Uncertainty caused by temperature variation

The temperature is recorded every two hours. Therefore, variations in an interval of two hours were calculated from 24 measurements and were found to be $0,06{ }^{\circ} \mathrm{C}$. Since these variations were cyclic, U-shaped distribution was used to calculate the standard uncertainty:

$$
u\left(\theta_{2}\right)=0,06 / \sqrt{2}=0,042^{\circ} \mathrm{C}
$$

- Total uncertainty $u(\bar{t})$

$$
u(\Delta \bar{t})=\sqrt{u\left(\theta_{1}\right)^{2}+u(\Delta \theta)^{2}+u\left(\theta_{2}\right)^{2}}=0,053^{\circ} \mathrm{C}
$$

7.2.9 Uncertainty of the correction for non-central contacting of the measuring faces of the measured $G B u\left(\delta L_{V}\right)$

For gauge blocks of grade 0 , the variation in length determined from measurements at the centre and the four corners has to be within $\pm 0,12 \mu \mathrm{m}$ (ISO $3650,1998)$. Assuming that this variation occurs on the measuring faces along the short edge of length $9 \mathrm{~mm}$ and that the central length is measured inside a circle of radius $0,5 \mathrm{~mm}$, the deviation due to central misalignment of the contacting point is estimated to be within an interval of $\pm 7 \mathrm{~nm}$. Standard uncertainty at supposed rectangular distribution is therefore:

$$
u\left(\delta L_{v}\right)=(7 \mathrm{~nm}) / \sqrt{3}=4,0 \mathrm{~nm}
$$

\subsection{Combined and Extended Standard Uncertainty of the Measurement}

By (7), combined standard uncertainty of the measurement was calculated to be:

$$
u_{s}=\sqrt{(16,8 \mathrm{~nm})^{2}+\left(0,25 \cdot 10^{-6} \cdot L\right)^{2}}
$$


Rounded expanded uncertainty of the measurement at $k=2$ is therefore:

$$
U=\sqrt{(35 \mathrm{~nm})^{2}+\left(0,5 \cdot 10^{-6} \cdot L\right)^{2}} ; k=2
$$

Calculated uncertainties are very low, also in comparison with CMCs of world best calibration institutes (national measurement institutes, NMI), as reported in key comparison database at BIPM $(* * * a)$.

\begin{tabular}{|c|c|c|c|c|c|}
\hline $\begin{array}{c}\text { Quantity } \\
X_{i}\end{array}$ & $\begin{array}{c}\text { Evaluated } \\
\text { value }\end{array}$ & Standard uncertainty & $\begin{array}{c}\text { Distri- } \\
\text { bution }\end{array}$ & $\begin{array}{c}\text { Sensitivity } \\
\text { coefficient }\end{array}$ & Uncertainty contribution \\
\hline$L_{S}$ & $100 \mathrm{~mm}$ & $\sqrt{(10 \mathrm{~nm})^{2}+\left(0,09 \cdot 10^{-6} \cdot L\right)^{2}}$ & normal & 1 & $\sqrt{(10 \mathrm{~nm})^{2}+\left(0,09 \cdot 10^{-6} \cdot \mathrm{L}\right)^{2}}$ \\
\hline$\delta L_{D}$ & $0 \mathrm{~mm}$ & $0,082 \cdot 10^{-6} \cdot L$ & triang. & 1 & $0,082 \cdot 10^{-6} \cdot L$ \\
\hline$\delta L$ & $0 \mathrm{~nm}$ & $\sqrt{(10,5 \mathrm{~nm})^{2}+\left(0,17 \cdot 10^{-6} \cdot L\right)^{2}}$ & normal & 1 & $\sqrt{(10,5 \mathrm{~nm})^{2}+\left(0,17 \cdot 10^{-6} \cdot \mathrm{L}\right)^{2}}$ \\
\hline$\delta L_{C}$ & $0 \mathrm{~mm}$ & negligible & normal & 1 & negligible \\
\hline $\bar{\alpha}$ & $\begin{array}{c}11,5 \cdot 10^{-6} \\
{ }^{\circ} \mathrm{C}^{-1}\end{array}$ & $0,289 \cdot 10^{-6}{ }^{\circ} \mathrm{C}^{-1}$ & rectang. & $-0,02{ }^{\circ} \mathrm{C} \cdot L$ & $-0,006 \cdot 10^{-6} \cdot L$ \\
\hline$\delta t$ & $0{ }^{\circ} \mathrm{C}$ & $0,0115^{\circ} \mathrm{C}$ & rectang. & $\begin{array}{c}-11,5 \cdot 10^{-6} \\
{ }^{\circ} \mathrm{C}^{-1} \cdot L\end{array}$ & $-0,132 \cdot 10^{-6} \cdot L$ \\
\hline$\delta \alpha$ & $0{ }^{\circ} \mathrm{C}^{-1}$ & $0,41 \cdot 10^{-6}{ }^{\circ} \mathrm{C}^{-1}$ & triang. & $\begin{array}{c}-0,05 \\
{ }^{\circ} \mathrm{C} \cdot L\end{array}$ & $-0,021 \cdot 10^{-6} \cdot L$ \\
\hline$\Delta \overline{\mathrm{t}}$ & $0{ }^{\circ} \mathrm{C}$ & $0,053{ }^{\circ} \mathrm{C}$ & normal & $\begin{array}{c}-1 \cdot 10^{-6}{ }^{\circ} \mathrm{C}^{-} \\
{ }^{1} \cdot L\end{array}$ & $-0,053 \cdot 10^{-6} \cdot L$ \\
\hline$\delta l_{v}$ & $0 \mathrm{~nm}$ & $4 \mathrm{~nm}$ & normal & 1 & $4 \mathrm{~nm}$ \\
\hline
\end{tabular}

Tab. 2. Standard uncertainties of the input value estimations and combined standard uncertainty

\section{Conclusion}

Gauge block calibration by mechanical comparison, as a secondary option for highest-level gauge blocks calibration, is inferior to interferometric only in increased uncertainty of the results. However, its instrumentation is less expensive and its procedure much simpler and faster, that is why it is widely used in calibration laboratories throughout the engineering industry.

As a national metrology laboratory for length, not performing interferometric gauge block calibration, we were handicapped by non-capability of accredited calibrating gauge blocks of dissimilar materials. 
After extensive experimental research considering stylus penetration, as well as thorough analytical approach, we succeeded in minimizing calibration uncertainty for the case of comparison of dissimilar materials. Procedure was already successfully accredited and entered as additional Calibration and measurement capability - CMC into the key comparison database at BIPM. Next research step is minimizing the calibration uncertainty of gauge block comparison of dissimilar materials.

\section{Acknowledgements}

Research was co-funded by Metrology Institute of the Republic of Slovenia (MIRS), as a part of co-funding of activities of the holder of the national standard (NMI) for length.

\section{References}

Acko, B. (2012). Calibration procedures with measurement uncertainty for advanced length standards and instruments. DAAAM International Publishing, ISBN 978-3901509-59-9, Vienna

EA-4/02 (1999). Expressions of the Uncertainty of Measurements in Calibration, European Accreditation

Faust, B.; Stoup, J. \& Stanfield, E. (1998). Minimizing Error Sources in Gage Block Mechanical Comparison Measurements. In: Proc. of SPIE, Vol. 3477, 127-136

ISO 3650 (1998): Geometrical product specifications (GPS) - Length standards Gauge blocks, International Organization for Standardization

ISO Guide to the expression of uncertainty in measurement (1995). International Organisation for Standardisation

Godina, A.; Acko, B. \& Drnovsek, J. (2006). Stylus penetration correction for the calibration of gauge blocks with dissimilar materials by mechanical comparison, Strojarstvo 48, 149-156

Godina, A.; Acko, B. \& Druzovec, M. (2007). New approach to uncertainty evaluation in the calibration of gauge block comparators, Measurement 40, 607-614 Godina, A.; Tasic, T. \& Acko, B. (2010). Uncertainty of mechanical calibration of gauge blocks of dissimilar materials. In: DAAAM International scientific book 2010, 431-444, Katalinic, B. (Ed.), DAAAM International Publishing, ISBN 978-3-90150969-8, Vienna

Mahr (1995). Betriebsanleitung : Endmaßgerät 826. Mahr GmbH, Göttingen

Thalmann, R. \& Baechler, H.(2003). Issues and advantages of gauge block calibration by mechanical comparison. In: Proc. of SPIE, Vol. 5190, 62-69

***a http://kcdb.bipm.org/AppendixC/country_list.asp?Iservice=L/DimMet.2.3.7 BIPM Key Comparison Database, Appendix C: Calibration and Measurement Capabilities of National Metrology Institutes, Length. Accesed on: 2013-08-01 